\title{
Geochemical Characteristics of Natural Gas and Hydrocarbon Charge History in the Western Qaidam Basin, Northwest China
}

\author{
Kefei Chen, ${ }^{1,2,3}$ Shixin Zhou $\mathbb{D}^{1,2}$ Jing Li $\mathbb{D}^{1,4}$ Chen Zhang, ${ }^{1,2,3}$ Zexiang Sun, ${ }^{1,2,3}$ \\ Pengpeng Li, ${ }^{1,2,3}$ and Bingkun Meng ${ }^{1,2,3}$ \\ ${ }^{1}$ Northwest Institute of Eco-Environment and Resources, Chinese Academy of Sciences, Lanzhou 730000, China \\ ${ }^{2}$ Key Laboratory of Petroleum Resources, Chinese Academy of Sciences, Lanzhou 730000, China \\ ${ }^{3}$ University of Chinese Academy of Sciences, Beijing 100049, China \\ ${ }^{4}$ Shandong Provincial Key Laboratory of Depositional Mineralization \& Sedimentary Mineral, Shandong University of Science \\ and Technology, Qingdao 266590, China
}

Correspondence should be addressed to Shixin Zhou; sxzhou@lzb.ac.cn and Jing Li; lj1926@lzb.ac.cn

Received 8 October 2019; Revised 20 February 2020; Accepted 23 May 2020; Published 1 August 2020

Academic Editor: Agnes Mazot

Copyright ( $(2020$ Kefei Chen et al. This is an open access article distributed under the Creative Commons Attribution License, which permits unrestricted use, distribution, and reproduction in any medium, provided the original work is properly cited.

31 natural gases in the western Qaidam Basin of China were collected and analyzed for gas composition including light hydrocarbons $\left(\mathrm{C}_{5}-\mathrm{C}_{7}\right)$ and carbon isotopic characteristics. Based on genetic type obtained from $\mathrm{C}_{1}-\mathrm{C}_{3}$ and $\mathrm{C}_{7}$ fractions, four types of gases are identified: oil-type gas, coal-type gas, biodegraded gas, and mixed gas. The oil-type gas is the predominanttype gas in the western Qaidam Basin; coal-type gas is mainly distributed in the Zhahaquan and Nanyishan fields; mixed gas is mainly in the Zhahaquan, Wunan, and Nanyishan fields; and biodegraded gas is mainly distributed in the Huatugou and Yuejinerhao fields. According to the empirical relationship between $\delta^{13} \mathrm{C}_{1}$ and the equivalent vitrinite reflectance $\left(R_{o}\right.$, \%) of source rock, the $R_{o}$ values of gas range from $0.6 \%$ to $1.5 \%$, with an average value of $0.9 \%$. The generation temperatures of major reservoired hydrocarbons (GTMRH) calculated from the $\mathrm{C}_{7}$ components range from $115.6^{\circ} \mathrm{C}$ to $141.7^{\circ} \mathrm{C}$, with an average value of $126.5^{\circ} \mathrm{C}$. These two maturity indicators have relatively positive correlation and reveal that the maturity of gas increases from west to east in the southwestern Qaidam Basin. Moreover, combining GTMRH with the homogenous temperature of petroleum inclusions, it is inferred that major petroleum charge in the western Qaidam Basin mainly occurred during the late period of the Himalayan movement. Deep hydrocarbon fluid sources were found in the Shizigou, Yingdong, Zhahaquan, and Nanyishan fields; thus, the deep reservoirs of paleouplifts adjacent to the hydrocarbon-generating depressions are estimated as a favorable area for further exploration in the western Qaidam Basin.

\section{Introduction}

The Qaidam Basin is the largest petroliferous sedimentary basin inside the Tibetan Plateau, northwest China, and significant amounts of oil have been produced in the western Qaidam Basin [1]. In recent years, oil and gas exploration has made considerable progress in the Yingdong, Zhahaquan, and Yingxi fields in the western Qaidam Basin. Some newly produced oil has lower viscosity and density with a higher gas-oil ratio, suggesting that they may be derived from deeper strata. However, little research has been conducted to investigate the genetic type and maturity of these newly produced gases. Furthermore, a systematic analysis of natural gas is necessary to deepen the recognition of gas origin and charge history in the western Qaidam Basin.

The hydrocarbon components of natural gas mainly consist of $\mathrm{C}_{1}-\mathrm{C}_{5}$ compounds with trace $\mathrm{C}_{5+}$ light hydrocarbon compounds. The definition of light hydrocarbon has not been unified. In early studies, light hydrocarbons refer to hydrocarbons ranging from $\mathrm{C}_{1}$ to $\mathrm{C}_{14}$ [2-4]; in later studies, the ones refer to hydrocarbons ranging from $\mathrm{C}_{5}$ to $\mathrm{C}_{10}$ [5-7]. The $\mathrm{C}_{5}-\mathrm{C}_{7}$ light hydrocarbons are the focus in current researches and this paper. Besides, partial light hydrocarbons would dissolve in the gas phase because natural gas can be taken as the solvent and the volatility of light hydrocarbon. Thus, light hydrocarbons could distribute in the gas phase 
and liquid phase in a gas sample. Previous studies reported that separation of the oil phase and the gas phase mainly affects the aromatic content of a light hydrocarbon, with little effect on most light hydrocarbon parameters [2, 8, 9]. So far, light hydrocarbons in dry gas and wet gas have been successfully analyzed [6,7]. Many studies have applied the light hydrocarbon as an ancillary geochemical tool to evaluate the genetic type and thermal maturity of natural gas and identify secondary alteration [3-7]. Furthermore, natural gas in western Qaidam Basin is wet gas with a higher content of light hydrocarbons, which could better reflect the geochemical characteristics of natural gas.

Identification of the genetic type of natural gas is important for assessment of its sources and exploration potential [10]. Based on sources, there are two broad categories: biogenic and abiogenic gases. Abiogenic gas includes gas from the mantle and abiogenic formation. Biogenic gas includes biogenic gas and thermogenic gas, and the latter can be further divided into coal-type gas and oil-type gas based on the type of organic matter $[11,12]$. Many researchers have already established some geochemical parameters and classic diagrams to identify the genetic type of natural gas [12-16]. The composition of natural gas and methane carbon isotope values $\left(\mathrm{C}_{1} /\left(\mathrm{C}_{2}+\mathrm{C}_{3}\right)\right.$ vs. $\left.\delta^{13} \mathrm{C}_{1}\right)$ is significant in identifying different types of natural gas [13]. Besides, the carbon isotope of ethane is widely used to distinguish the coal-type gas and the oil-type gas [14], including the cross plot of $\delta^{13} \mathrm{C}_{1}-\delta^{13} \mathrm{C}_{2}$ $\delta^{13} \mathrm{C}_{3}, \delta^{13} \mathrm{C}_{1}-\delta^{13} \mathrm{C}_{\mathrm{CO} 2}, \delta^{13} \mathrm{C}_{2-3}-\ln \left(\mathrm{C}_{2} / \mathrm{C}_{3}\right)$, and so on $[12,15$, 16]. Natural gas in the western Qaidam Basin is mainly derived from saline lacustrine depositions which are enriched in $\delta^{13} \mathrm{C}_{2}$, and Zhang et al. [17] modified the classification criteria for coal-type gas and oil-type gas. Owing to the complex formation process of natural gas and secondary alteration, the genetic identification of different types of natural gas should be based on multiple parameters. $C_{5}-C_{7}$ light hydrocarbons can be used to provide some new information insight into natural gas generation. Therefore, a detailed study of natural gas could get a better understanding of its genetic type.

Thermal maturity has an important effect on the composition and carbon isotope of natural gas. The carbon isotope values of methane increase with increasing thermal maturity of source rocks and are adapted to study the maturity of gas $[18,19]$. Besides, the dryness coefficient of natural gas increases with an increase of thermal maturity. Moreover, light hydrocarbons could be used to calculate the expulsion temperature of oil and gas [20]. The ratio of 2,4-dimethylpentane to 2,3-dimethylpentane $\left(2,4-\mathrm{DMC}_{5} / 2,3-\mathrm{DMC}_{5}\right)$ was found related to temperature, and it was calibrated to expulsion temperature $[8,21]$. Studies have reported that expulsion temperatures have positive correlations with biomarker maturity parameters and the gas-oil ratio [22-24]. While little research has been conducted to evaluate the expulsion temperatures of natural gas in the western Qaidam Basin, it may provide a novel way of assessing the maturity of gas.

The homogenous temperatures of petroleum inclusions are always applied to reconstruct the petroleum charging history combining with burial history and hydrocarbon generation history. Numerous researches have been carried out, and most suggest that there are primarily two petroleum charging episodes in the western Qaidam Basin [25-30]. Because each hydrocarbon charge event has distinct contributions to reservoired petroleum, it is important to recognize that a major charge event is not only of academic significance but also related directly to the evaluation of the commercial potential in a studied area. Dieckmann et al. [20] proposed that expulsion temperature calculated from the $\mathrm{C}_{7}$ light hydrocarbon could reflect the average temperature of hydrocarbon expulsion. Generally, the reservoired petroleum is composed of hydrocarbons from multiperiod charging and dominated by the ones from the major period, so that the expulsion temperature obtained from the $\mathrm{C}_{7}$ light hydrocarbon, which is collected from reservoired petroleum, could be considered as the generation temperature of major reservoired hydrocarbons (GTMRH). The GTMRH reflects the character of reservoired petroleum and may provide information about major petroleum charging.

In this study, 31 natural gases in the western Qaidam Basin of China were collected and analyzed for gas composition and carbon isotopic characteristics. The aims of this study are (1) to determine the genetic type and thermal maturity of natural gas in the western Qaidam Basin, (2) to investigate the application of GTMRH to petroleum charging history, and (3) to provide suggestions for future hydrocarbon exploration.

\section{Geological Setting}

The Qaidam Basin is located in the northeastern Tibetan Plateau of northwest China and covers a total area of $121,000 \mathrm{~km}^{2}$ (Figure 1(a)). It contains Mesozoic and Cenozoic lacustrine sedimentary sequences that were deposited on the Qaidam block during the pre-Jurassic period, and maximum sediment thickness could reach 17,280 m [1]. The basin is bounded by the Qilian Mountains to the northeast, the Altun Mountains to the northwest, and the Kunlun Mountains to the south. The basin can be structurally divided into three tectonic units, namely, the Northern Fault-Block Belt, the Western Depression, and the Eastern Depression. This study was carried out in the Western Depression (Figure 1(b)). Besides, the western Qaidam Basin includes two parts: the northwestern and the southwestern Qaidam Basin, and gas samples in both parts are obtained in this study (Figure 1(c)).

In the western Qaidam Basin, the Tertiary saline lacustrine depositions are primary hydrocarbon source rocks. The source rocks are lacustrine mudstone and marlstone in the Lower Xiaganchaigou-Shangganchaigou Formations. The main reservoirs are sandstone in the Lulehe-Shangyoushashan Formations and marlstone in the Upper Xiaganchaigou Formation. The cap rocks are mudstone in the Lulehe-Shizigou Formations and marlstone in the Upper Xiaganchaigou Formation (Figure 2).

Source rocks in the western Qaidam Basin have relatively low amount of total organic carbon (TOC), 0.1-2.7\% (average below $1.0 \%$ ), but their generation potentials reach a fairly high level with chloroform extraction mainly between $0.05 \%$ and $0.5 \%$ [31]. The organic matter of two intervals is 


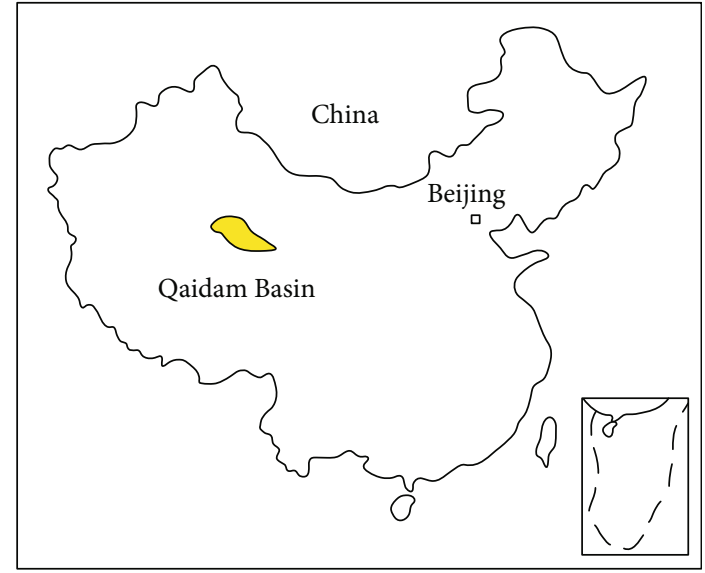

(a)

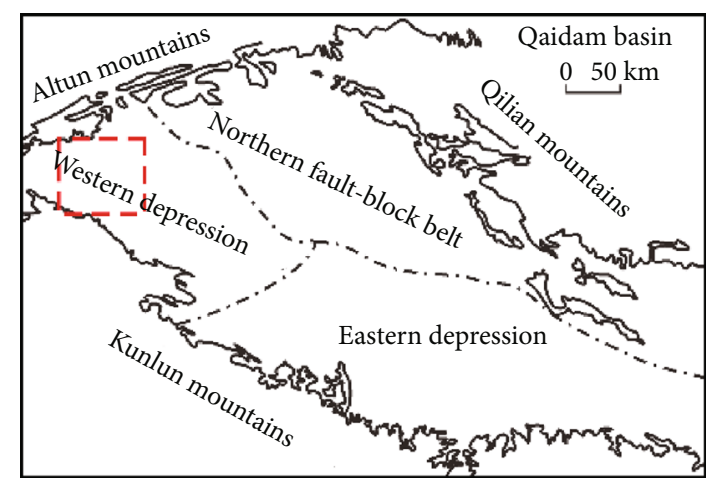

(b)

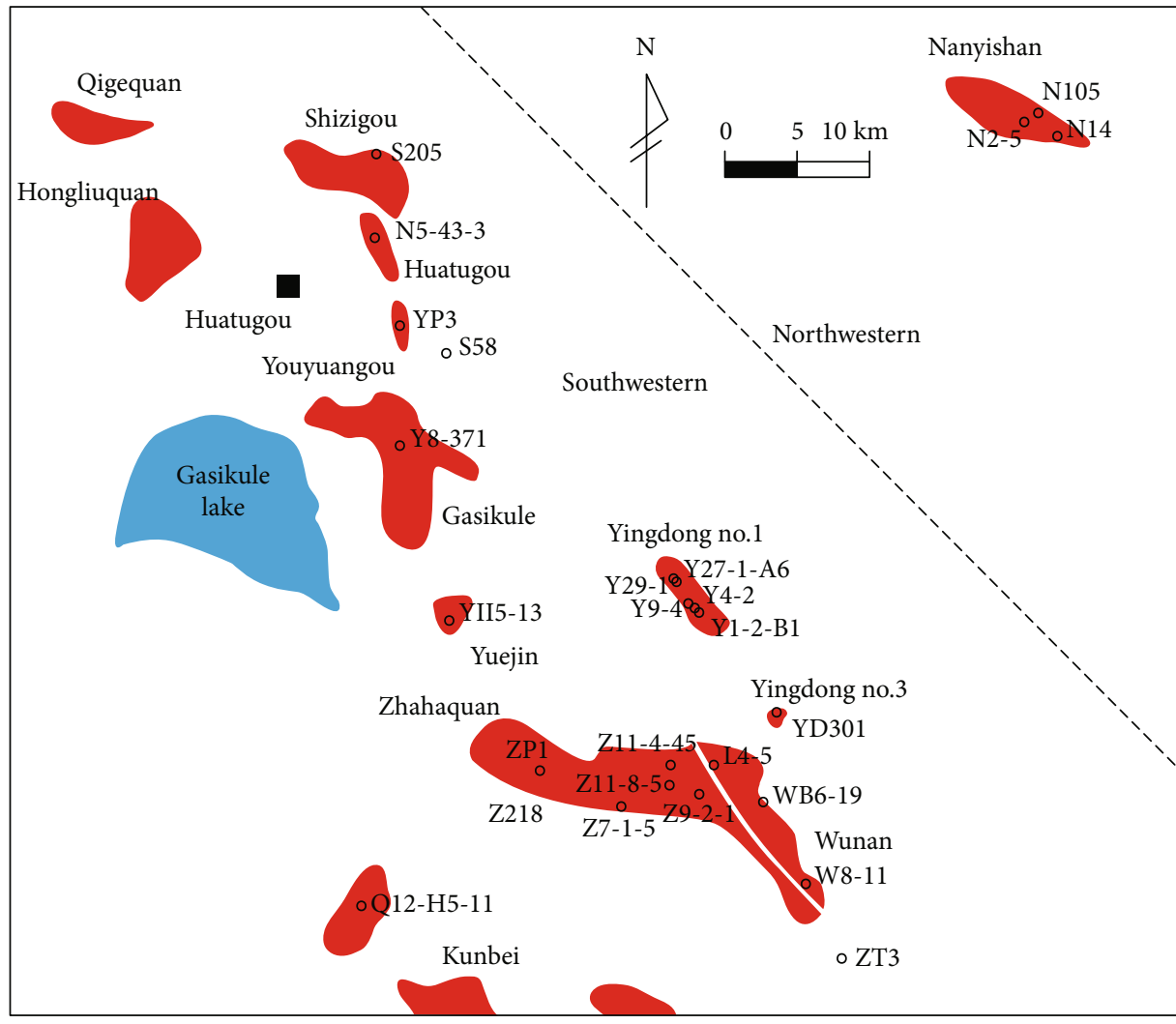

Tectonic unit boundary

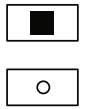

Resident site

Well

(c)

FIGURE 1: (a) Location of the Qaidam Basin within China; (b) location of three tectonic units in the Qaidam Basin and the rectangle indicates the studied area; (c) distribution of oil fields and sample sites and the dashed line is the boundary between southwestern and northwestern Qaidam Basin.

mainly type-II kerogen and a little of type-III kerogen [32]. The vitrinite reflectance values $\left(R_{o}, \%\right)$ of these source rocks range from $0.4 \%$ to $1.3 \%$ [31]; corresponding oils within the immature to mature stages are all discovered in the western Qaidam Basin [33, 34].

\section{Samples and Methods}

3.1. Sampling. Natural gases were sampled in the major oil fields, e.g., Shizigou, Huatugou, Youyuangou, Gasikule, Yuejinerhao, Yingdong (including no. 1 and no. 3), Wunan, 


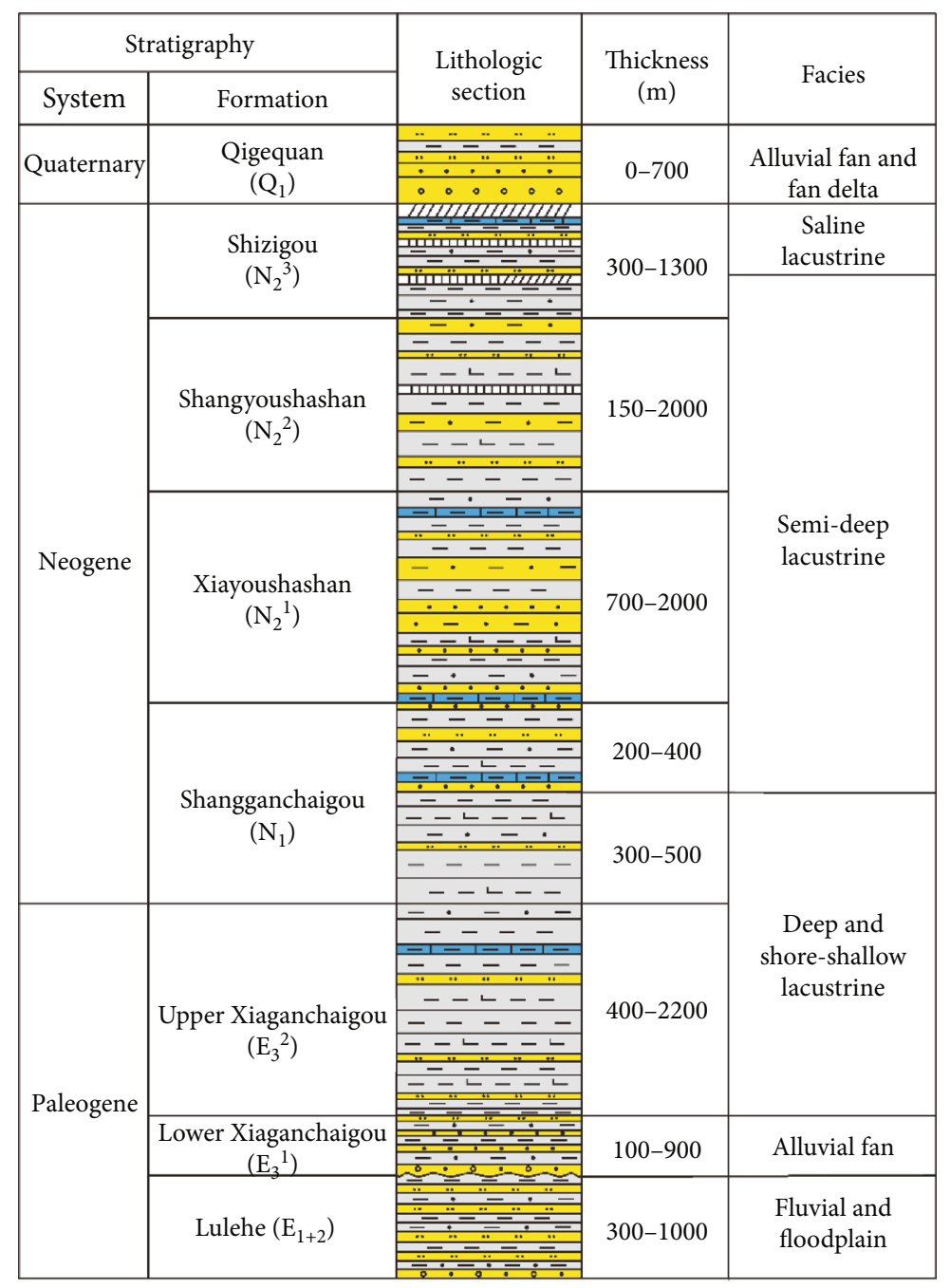

\begin{tabular}{|c|c|}
\hline $\begin{array}{ll}\cdot . \cdot \\
\end{array}$ & Siltstone \\
\hline$\cdot \cdot$ & Conglomerate sandstone \\
\hline-- & Mudstone \\
\hline$-\div-$ & Calcareous mudstone \\
\hline 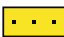 & Sandstone \\
\hline
\end{tabular}

\begin{tabular}{|c|c|}
\hline $1-$ & Muddy limestone \\
\hline IIسय & Salt rock \\
\hline & Sandy mudstone \\
\hline Iسm & Gypsum \\
\hline$\cdot-\cdot$ & Muddy sandstone \\
\hline
\end{tabular}

Figure 2: Generalized stratigraphic column for the western Qaidam Basin (modified after [46]).

Zhahaquan, Kunbei, and Nanyishan oil fields (Figure 1(c)). Gas samples were directly collected at wellheads in the commercial oil production fields using aluminum alloy cylinders with double valves. Before sampling, cylinders were flushed three times at the wellhead to remove air. In total, 31 natural gases were sampled in the western Qaidam Basin, and the sample data are listed in Table 1.

Because of the volatility of light hydrocarbons, evaporation is inevitable during sampling, storage, and sample preparation when using crude oil to analyze light hydrocarbon composition, and even minor evaporation will affect light hydrocarbon parameters [35], while natural gas stored in aluminum alloy cylinders has no evaporation, which is essential for the original light hydrocarbon component.
3.2. Gas Composition and Stable Carbon Isotopic Analysis. Gas compositions were determined on a GC-9160 gas chromatograph equipped with two flame ionization detectors and a thermal conductivity detector. Individual hydrocarbon gas components from $\mathrm{C}_{1}$ to $\mathrm{C}_{5}$ were separated using a capillary column (PLOT $\mathrm{Al}_{2} \mathrm{O}_{3} 50 \mathrm{~m} \times 0.53 \mathrm{~mm} \times 0.32 \mu \mathrm{m}$ ). The GC oven temperature was initially held at $35^{\circ} \mathrm{C}$ for $5 \mathrm{~min}$ and then increased to $200^{\circ} \mathrm{C}$ at $10^{\circ} \mathrm{C} / \mathrm{min}$ and held at this temperature for $10 \mathrm{~min}$. Gaseous nonhydrocarbons $\left(\mathrm{N}_{2}, \mathrm{CO}_{2}\right.$, and $\left.\mathrm{H}_{2} \mathrm{~S}\right)$ were analyzed on a high-resolution mass spectrometer with an electric impact ion source, using a selected ion-monitoring method to determine molar percentages, which were converted to molar concentration using the ideal gas function. The emission current was $40 \mu \mathrm{A}$, and 
TABLE 1: Representative light hydrocarbon geochemical parameters in natural gas from the western Qaidam Basin.

\begin{tabular}{|c|c|c|c|c|c|c|c|c|c|c|}
\hline Field & Well & Depth (m) & Strata & $\begin{array}{l}n-\mathrm{C}_{7} / \\
\mathrm{MCC}_{6}\end{array}$ & $\begin{array}{c}\text { Toluene/ } \\
n-\mathrm{C}_{7}\end{array}$ & $\begin{array}{c}\Sigma \mathrm{DMCC}_{5} \\
(\%)\end{array}$ & $\begin{array}{c}n-\mathrm{C}_{7} \\
(\%) \\
\end{array}$ & $\begin{array}{c}\mathrm{MCC}_{6} \\
(\%)\end{array}$ & $\begin{array}{c}2-\mathrm{MC}_{6} / \\
3-\mathrm{MC}_{6}\end{array}$ & $\begin{array}{c}\text { GTMRH } \\
\left({ }^{\circ} \mathrm{C}\right)\end{array}$ \\
\hline \multirow{2}{*}{ Shizigou } & S205 & $3378-3598$ & $\mathrm{E}_{3}^{2}$ & 1.69 & 0.40 & 18.0 & 51.5 & 30.5 & 0.74 & 115.6 \\
\hline & S58 & 5451.18 & $\mathrm{E}_{3}^{2}$ & 1.23 & 0.74 & 20.2 & 44.0 & 35.7 & 0.72 & 119.5 \\
\hline Huatugou & HN5-43-3 & 1015 & $\mathrm{~N}_{1}$ & 1.73 & 0.10 & 30.4 & 44.1 & 25.5 & 0.83 & 121.8 \\
\hline Youyuangou & YP3 & $782.0-783.7$ & $\mathrm{~N}_{2}{ }^{1}$ & 2.72 & 0.19 & 13.9 & 63.0 & 23.1 & 1.12 & 128.5 \\
\hline Gasikule & Y8-371 & $3481.8-3528.0$ & $\mathrm{E}_{3}^{2}$ & 2.41 & 0.23 & 15.9 & 59.5 & 24.7 & 0.86 & 124.3 \\
\hline Yuejinerhao & YII5-13 & - & $\mathrm{E}_{3}{ }^{1}$ & 1.13 & 0.49 & 40.3 & 31.7 & 28.0 & 0.70 & 126.4 \\
\hline \multirow{7}{*}{$\begin{array}{l}\text { Yingdong } \\
\text { no. } 1\end{array}$} & Y1-2-B1 & $966-996.1$ & $\mathrm{~N}_{2}^{2}$ & 1.78 & 0.58 & 19.3 & 51.7 & 29.1 & 1.18 & 126.9 \\
\hline & Y1-2-B4 & $1288.3-1318.1$ & $\mathrm{~N}_{2}^{2}$ & 2.66 & 0.41 & 17.8 & 59.7 & 22.5 & 1.12 & 128.4 \\
\hline & Y27-1-A6 & 1739-1802 & $\mathrm{N}_{2}^{2}$ & 2.81 & 0.36 & 15.7 & 62.2 & 22.2 & 1.41 & 130.4 \\
\hline & Y38-3-C & $1271.5-1342.9$ & $\mathrm{~N}_{2}^{2}$ & 2.60 & 0.53 & 20.5 & 57.4 & 22.1 & 1.19 & 127.3 \\
\hline & Y29-1 & $2838.2-2896.8$ & $\mathrm{~N}_{2}{ }^{1}$ & 2.16 & 0.62 & 20.7 & 54.1 & 25.1 & 1.00 & 124.4 \\
\hline & Y4-2 & $3018.7-3103.7$ & $\mathrm{~N}_{2}{ }^{1}$ & 2.32 & 0.36 & 16.9 & 58.0 & 25.0 & 1.02 & 127.4 \\
\hline & Y9-4 & $2081.3-2219.9$ & $\mathrm{~N}_{2}{ }^{1}$ & 2.68 & 0.39 & 21.0 & 57.5 & 21.5 & 1.38 & 132.1 \\
\hline \multirow{3}{*}{$\begin{array}{l}\text { Yingdong } \\
\text { no. } 3\end{array}$} & YD301 & $2012.2-2023.3$ & $\mathrm{~N}_{2}{ }^{1}$ & 2.14 & 0.56 & 20.5 & 54.2 & 25.3 & 1.08 & 124.2 \\
\hline & YD3-3 & 2313.3-2491.4 & $\mathrm{N}_{2}^{1}$ & 2.22 & 0.50 & 20.7 & 54.6 & 24.7 & 1.16 & 133.3 \\
\hline & L4-5 & $2041.6-2305.5$ & $\mathrm{~N}_{2}^{1}$ & 1.89 & 2.65 & 16.3 & 54.7 & 28.9 & 1.09 & 121.4 \\
\hline \multirow[t]{6}{*}{ Wunan } & W8-11 & $1361.7-1420.7$ & $\mathrm{~N}_{2}^{1}$ & 1.60 & 0.77 & 10.1 & 55.3 & 34.5 & 1.01 & 130.2 \\
\hline & WB6-19 & $1631.8-1734$ & $\mathrm{~N}_{2}{ }^{1}$ & 1.37 & 0.49 & 18.0 & 47.4 & 34.6 & 0.81 & 121.3 \\
\hline & Z11-33-5 & - & $\mathrm{N}_{2}{ }^{1}$ & 2.61 & 0.22 & 20.1 & 57.8 & 22.2 & 1.11 & 125.6 \\
\hline & Z11-4-45 & - & $\mathrm{N}_{2}{ }^{1}$ & 2.51 & 0.20 & 20.6 & 56.7 & 22.6 & 1.11 & 125.7 \\
\hline & Z11-8-5 & $2493.0-2494.1$ & $\mathrm{~N}_{2}{ }^{1}$ & 2.04 & 0.57 & 19.7 & 53.9 & 26.4 & 1.00 & 127.2 \\
\hline & Z218 & - & $\mathrm{N}_{2}^{1}$ & 2.71 & 0.39 & 46.9 & 38.8 & 14.3 & 0.92 & 129.3 \\
\hline \multirow[t]{5}{*}{ Zhahaquan } & Z7-1-5 & $3530.8-3534.7$ & $\mathrm{~N}_{1}$ & 2.28 & 0.29 & 14.7 & 59.3 & 26.0 & 1.00 & 129.1 \\
\hline & $\mathrm{Z7}-2-3$ & - & $\mathrm{N}_{1}$ & 2.28 & 0.39 & 15.1 & 59.0 & 25.8 & 1.11 & 130.1 \\
\hline & Z9-2-1 & $2432.2-2840.6$ & $\mathrm{~N}_{2}^{1}$ & 1.85 & 0.51 & 19.6 & 52.2 & 28.2 & 1.00 & 125.1 \\
\hline & $\mathrm{ZP} 1$ & - & $\mathrm{N}_{2}{ }^{1}$ & 1.93 & 0.37 & 22.6 & 51.0 & 26.5 & 0.92 & 128.3 \\
\hline & $\mathrm{ZT} 3$ & $3144-3150$ & $\mathrm{E}_{3}{ }^{1}$ & 8.77 & 0.29 & 5.7 & 84.6 & 9.6 & 1.10 & 141.7 \\
\hline \multirow[t]{2}{*}{ Kunbei } & Q12-H5-11 & - & $\mathrm{E}_{3}{ }^{1}$ & 1.78 & 0.06 & 47.8 & 33.5 & 18.8 & 1.03 & 117.7 \\
\hline & N105 & $2342.3-2346.6$ & $\mathrm{~N}_{2}{ }^{1}$ & 0.75 & 1.05 & 19.4 & 34.6 & 46.0 & 0.96 & 124.2 \\
\hline \multirow[t]{2}{*}{ Nanyishan } & N14 & $4358-4457$ & $\mathrm{E}_{3}^{2}$ & 1.32 & 1.74 & 13.3 & 49.4 & 37.4 & 0.84 & - \\
\hline & $\mathrm{N} 2-5$ & - & $\mathrm{E}_{3}^{2}$ & 1.67 & 0.31 & 19.5 & 50.4 & 30.1 & 1.06 & 128.0 \\
\hline
\end{tabular}

$\Sigma \mathrm{DMCC}_{5}=\Sigma \mathrm{DMCC}_{5} \times 100 /\left(n-\mathrm{C}_{7}+\mathrm{MCC}_{6}+\Sigma \mathrm{DMCC}_{5}\right) ; n-\mathrm{C}_{7}=n-\mathrm{C}_{7} \times 100 /\left(n-\mathrm{C}_{7}+\mathrm{MCC}_{6}+\Sigma \mathrm{DMCC}_{5}\right) ; \mathrm{MCC}_{6}=\mathrm{MCC}_{6} \times 100 /\left(n-\mathrm{C}_{7}+\mathrm{MCC}_{6}+\Sigma \mathrm{DMCC}_{5}\right)$. GTMRH: generation temperature of major reservoired hydrocarbons; $T=140+15 \times \ln \left(2,4-\mathrm{DMC}_{5} / 2,3-\mathrm{DMC}_{5}\right)$ (Mango [8]).

the ionization energy of the ion source was $86 \mathrm{eV}$. The experimental error determined by this analysis approach for gas yields was $\pm 2 \%$ for each component.

Stable carbon isotope ratios were determined using a Finnigan MAT DELTAPlus mass spectrometer interfaced with a gas chromatograph. Gas components were separated on the gas chromatograph using helium as the carrier gas, converted into $\mathrm{CO}_{2}$ in a combustion interface and then introduced into the mass spectrometer. Individual hydrocarbon gas components $\left(\mathrm{C}_{1}-\mathrm{C}_{5}\right)$ and $\mathrm{CO}_{2}$ were initially separated using a CP-CarboBOND column $(50 \mathrm{~m} \times 0.53 \mathrm{~mm} \times 15 \mu \mathrm{m})$. The GC oven temperature was increased from $60^{\circ} \mathrm{C}$ to $200^{\circ} \mathrm{C}$ at $15^{\circ} \mathrm{C} / \mathrm{min}$ (held $20 \mathrm{~min}$ ). High purity methane (-28.5 $\pm 0.5 \%$, VPDB) is used as an internal standard for each sample test in order to test the stability and accuracy of the instrument. The precision was $\pm 0.5 \%$. Stable carbon isotopic compositions were presented as $\delta^{13} \mathrm{C}$ values relative to the VPDB scales.

3.3. Light Hydrocarbon Analysis. The light hydrocarbon composition was analyzed on an Agilent $6890 \mathrm{~N}$ gas chromatograph (GC) with a $5973 \mathrm{~N}$ mass spectrometer (MS). The GC was equipped with a split/splitless injector, a fused silica column $(100 \mathrm{~m} \times 0.25 \mathrm{~mm}$ i.d. $\times 0.5 \mu \mathrm{m}$ film thickness), and a flame ionization detector $\left(300^{\circ} \mathrm{C}\right)$. The injected sample volume was $1 \mathrm{ml}$ (split ratio set to $20: 1$ ) and the injector temperature was $150^{\circ} \mathrm{C}$. The oven temperature program was programed from $40^{\circ} \mathrm{C}(15 \mathrm{~min})$ to $120^{\circ} \mathrm{C}$ (held $20 \mathrm{~min}$ ) at $2^{\circ} \mathrm{C} / \mathrm{min}$ and then to $290^{\circ} \mathrm{C}$ (held $20 \mathrm{~min}$ ) at $12^{\circ} \mathrm{C} / \mathrm{min}$. Helium was used as the carrier gas (flow rate $1.0 \mathrm{ml} / \mathrm{min}$ ). The MS conditions were electron ionization at $70 \mathrm{eV}$ with an ion source temperature of $230^{\circ} \mathrm{C}$. Compounds were 


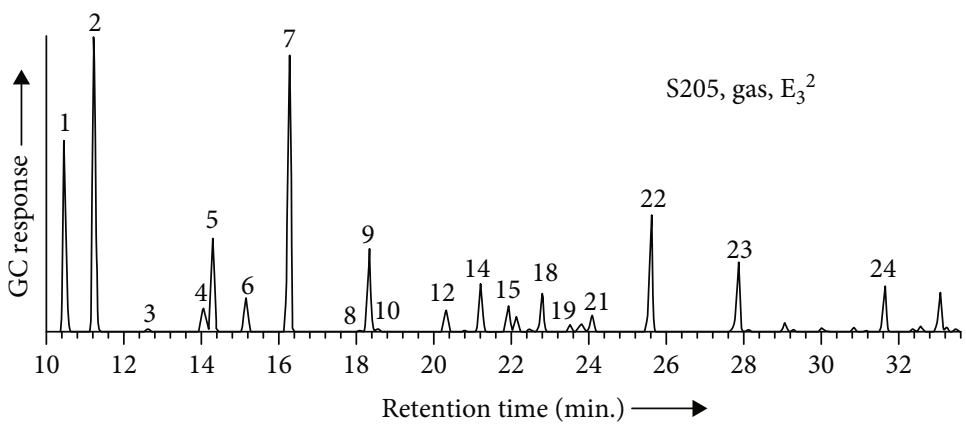

(a)

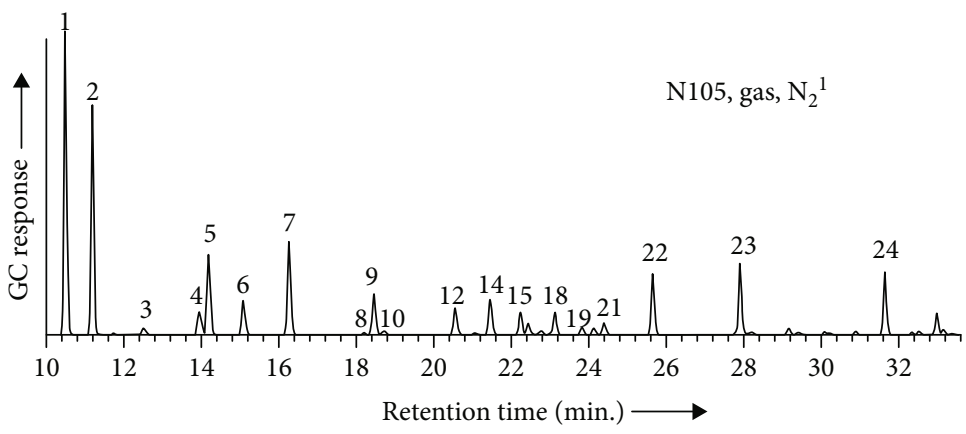

(b)

Figure 3: Representative gas chromatogram of light hydrocarbons $\left(\mathrm{C}_{5}-\mathrm{C}_{7}\right)$ in natural gas from the western Qaidam Basin. The numbers and the corresponding light hydrocarbons are listed in Table 2.

identified by matching mass spectra with the NIST library of standard compounds (Figure 3 and Table 2). The used light hydrocarbons were obtained from integrated peak areas on the gas chromatograms.

\section{Results}

4.1. Natural Gas Components. The composition of natural gas in the western Qaidam Basin is mainly composed of hydrocarbons (average 91.6\%). Nonhydrocarbon gases include nitrogen (average $4.2 \%$ ), carbon dioxide (average 1.2\%), and hydrogen sulfide (gas content in well S58 is 1.74\%). Among the hydrocarbons, methane content is $58.7-95.6 \%$, with an average of $81.0 \%$; ethane content is $0.2-17.4 \%$, with an average of $6.9 \%$; and propane content is $0.1-9.4 \%$, with an average of $1.9 \%$. The gas dryness coefficient $\left(\mathrm{C}_{1} / \mathrm{C}_{1-5}\right)$ is in the range of $0.66-0.99$, with an average of 0.88 , indicating that gases are mainly wet gases (Table 3 ).

4.2. Carbon Isotopes of Natural Gas. In the western Qaidam Basin, $\delta^{13} \mathrm{C}_{\text {methane }}$ values range from $-54.6 \%$ to $-28.6 \%$, with an average of $-38.9 \% ; \delta^{13} \mathrm{C}_{\text {ethane }}$ values range from $-35.9 \%$ o to $-20.5 \%$, with an average of $-26.9 \%$; and $\delta^{13} \mathrm{C}_{\text {propane }}$ values range from $-28.1 \%$ to $-20.7 \%$, with an average of $-24.3 \%$ o (Table 3). Almost all gases from the western Qaidam Basin have an expected carbon isotopic distribution pattern among the $\mathrm{C}_{1}-\mathrm{C}_{3}$ alkanes, i.e., $\delta^{13} \mathrm{C}_{1}<\delta^{13} \mathrm{C}_{2}<\delta^{13} \mathrm{C}_{3}$, suggesting that most natural gases are primary gases. Only the N14 gas sample from the Nanyishan field has partial carbon isotopic reversal between ethane and propane $\left(\delta^{13} \mathrm{C}_{2}>\delta^{13} \mathrm{C}_{3}\right)$, probably implying admixture of gases from various origins.
TABLE 2: Number and the corresponding light hydrocarbon.

\begin{tabular}{|c|c|c|}
\hline Number & Chemical name & Abbreviation \\
\hline 1 & $i-\mathrm{C}_{5}$ & $i-\mathrm{C}_{5}$ \\
\hline 2 & $n-\mathrm{C}_{5}$ & $n-\mathrm{C}_{5}$ \\
\hline 3 & 2,2-Dimethylbutane & $2,2-\mathrm{DMC}_{4}$ \\
\hline 4 & Cyclopentane & $\mathrm{CC}_{5}$ \\
\hline 5 & 2-Methylpentane & $2-\mathrm{MC}_{5}$ \\
\hline 6 & 3-Methylpentane & $3-\mathrm{MC}_{5}$ \\
\hline 7 & $n-\mathrm{C}_{6}$ & $n-\mathrm{C}_{6}$ \\
\hline 8 & 2,2-Dimethylpentane & $2,2-\mathrm{DMC}_{5}$ \\
\hline 9 & Methylcyclopentane & $\mathrm{MCC}_{5}$ \\
\hline 10 & 2,4-Dimethylpentane & $2,4-\mathrm{DMC}_{5}$ \\
\hline 11 & 2,2,3-Trimethylbutane & $2,2,3-\mathrm{TMC}_{4}$ \\
\hline 12 & Benzene & Benz. \\
\hline 13 & 3,3-Dimethylpentane & $3,3-\mathrm{DMC}_{5}$ \\
\hline 14 & Cyclohexane & $\mathrm{CC}_{6}$ \\
\hline 15 & 2-Methylhexane & $2-\mathrm{MC}_{6}$ \\
\hline 16 & 2,3-Dimethylpentane & $2,3-\mathrm{DMC}_{5}$ \\
\hline 17 & 1,1-Dimethylcyclopentane & $1,1-\mathrm{DMCC}_{5}$ \\
\hline 18 & 3-Methylhexane & $3-\mathrm{MC}_{6}$ \\
\hline 19 & cis-1,3-Dimethylcyclopentane & $1, \mathrm{c} 3-\mathrm{DMCC}_{5}$ \\
\hline 20 & trans-1,3-Dimethylcyclopentane & $1, \mathrm{t} 3-\mathrm{DMCC}_{5}$ \\
\hline 21 & trans-1,2-Dimethylcyclopentane & $1, \mathrm{t} 2-\mathrm{DMCC}_{5}$ \\
\hline 22 & $n-\mathrm{C}_{7}$ & $n-\mathrm{C}_{7}$ \\
\hline 23 & Methylcyclohexane & $\mathrm{MCC}_{6}$ \\
\hline 24 & Toluene & Tol. \\
\hline
\end{tabular}


TABLE 3: Component and isotope properties of natural gas in the western Qaidam Basin.

\begin{tabular}{|c|c|c|c|c|c|c|c|c|c|c|c|c|c|c|c|c|c|}
\hline \multirow{2}{*}{ Well } & \multicolumn{9}{|c|}{ Main components (\%) } & \multirow[b]{2}{*}{$\mathrm{H}_{2} \mathrm{~S}$} & \multirow{2}{*}{ Dryness } & \multirow{2}{*}{$\mathrm{C}_{1} /\left(\mathrm{C}_{2}+\mathrm{C}_{3}\right)$} & \multicolumn{3}{|c|}{$\delta^{13} \mathrm{C}(\%, \mathrm{VPDB})$} & \multirow{2}{*}{$R_{o}(\%)^{*}$} & \multirow{2}{*}{$R_{o}(\%)^{* *}$} \\
\hline & $\mathrm{C}_{1}$ & $\mathrm{C}_{2}$ & $\mathrm{C}_{3}$ & $i-\mathrm{C}_{4}$ & $n-C_{4}$ & $i-\mathrm{C}_{5}$ & $n-C_{5}$ & $\mathrm{CO}_{2}$ & $\mathrm{~N}_{2}$ & & & & $\mathrm{C}_{1}$ & $\mathrm{C}_{2}$ & $\mathrm{C}_{3}$ & & \\
\hline S205 & 79.49 & 6.41 & 4.37 & 0.94 & 2.08 & 0.86 & 0.93 & 0.60 & 3.02 & 0.00 & 0.84 & 7.4 & -40.6 & -29.8 & -26.4 & & 0.8 \\
\hline S58 & 78.77 & 5.98 & 3.54 & 0.79 & 1.92 & 0.76 & 0.99 & 1.44 & 2.74 & 1.74 & 0.85 & 8.3 & -40.0 & -27.7 & & & 0.9 \\
\hline HN5-43-3 & 67.54 & 5.97 & 2.42 & 0.40 & 0.74 & 0.24 & 0.26 & 1.61 & 16.87 & 0.00 & 0.87 & 8.1 & -54.6 & -35.9 & -28.1 & & 0.6 \\
\hline YP3 & 72.94 & 10.97 & 0.89 & 1.09 & 0.02 & 0.33 & 0.31 & 1.24 & 5.35 & 0.00 & 0.84 & 6.2 & -40.6 & -27.9 & -23.1 & & 0.8 \\
\hline Y8-371 & 80.09 & 5.77 & 0.47 & 0.69 & 0.00 & 0.28 & 0.32 & 2.18 & 6.59 & 0.00 & 0.91 & 12.8 & -42.1 & -28.4 & -26.8 & & 0.8 \\
\hline YII5-13 & 91.01 & 1.75 & 0.50 & 0.38 & 0.01 & 0.17 & 0.24 & 1.82 & 1.71 & 0.00 & 0.97 & 40.5 & -47.7 & -28.2 & -21.0 & & 0.7 \\
\hline Y1-2-B1 & 73.34 & 9.91 & 1.00 & 1.24 & 0.01 & 0.31 & 0.25 & 1.70 & 4.33 & 0.00 & 0.85 & 6.7 & -37.9 & -27.2 & -24.7 & & 0.9 \\
\hline Y1-2-B4 & 87.93 & 4.27 & 0.38 & 0.48 & 0.00 & 0.17 & 0.16 & 0.68 & 2.85 & 0.00 & 0.94 & 18.9 & -36.6 & -26.8 & -23.2 & & 0.9 \\
\hline Y27-1-A6 & 86.30 & 4.95 & 0.43 & 0.58 & 0.00 & 0.20 & 0.20 & 0.75 & 3.20 & 0.00 & 0.93 & 16.0 & -37.7 & -26.7 & -24.3 & & 0.9 \\
\hline Y38-3-C & 88.03 & 4.95 & 0.44 & 0.58 & 0.00 & 0.20 & 0.21 & 0.00 & 2.14 & 0.00 & 0.93 & 16.3 & -39.5 & -27.6 & -25.0 & & 0.9 \\
\hline Y29-1 & 81.66 & 6.29 & 0.60 & 0.74 & 0.01 & 0.19 & 0.19 & 1.38 & 4.10 & 0.00 & 0.91 & 11.8 & -40.5 & -30.9 & -25.6 & & 0.9 \\
\hline Y4-2 & 79.50 & 6.73 & 0.79 & 1.04 & 0.01 & 0.33 & 0.29 & 1.61 & 4.12 & 0.00 & 0.90 & 10.6 & -39.6 & -27.8 & -24.4 & & 0.9 \\
\hline Y9-4 & 81.61 & 3.59 & 0.60 & 0.79 & 0.01 & 0.28 & 0.26 & 0.70 & 7.22 & 0.00 & 0.94 & 19.5 & -40.0 & -28.7 & -27.4 & & 0.9 \\
\hline YD301 & 80.39 & 6.09 & 0.88 & 1.20 & 0.00 & 0.25 & 0.31 & 1.27 & 3.92 & 0.00 & 0.90 & 11.5 & -39.5 & -27.0 & -23.9 & & 0.9 \\
\hline YD3-3 & 79.96 & 6.63 & 0.68 & 1.01 & 0.01 & 0.41 & 0.40 & 1.78 & 4.37 & 0.00 & 0.90 & 10.9 & -40.5 & -26.8 & -24.3 & & 0.9 \\
\hline L4-5 & 80.76 & 7.14 & 0.64 & 0.89 & 0.01 & 0.31 & 0.42 & 1.15 & 3.64 & 0.00 & 0.90 & 10.4 & -39.5 & -27.0 & -25.0 & & 0.9 \\
\hline W8-11 & 87.32 & 6.20 & 2.51 & 0.41 & 0.60 & 0.17 & 0.17 & 0.09 & 2.17 & 0.00 & 0.90 & 10.0 & -28.6 & -24.3 & -23.9 & 1.5 & \\
\hline WB6-19 & 86.33 & 6.28 & 0.62 & 0.72 & 0.01 & 0.21 & 0.28 & 0.00 & 1.42 & 0.00 & 0.91 & 12.5 & -42.1 & -26.1 & -24.2 & & 0.8 \\
\hline Z11-33-5 & 88.05 & 5.24 & 2.35 & 0.46 & 0.74 & 0.27 & 0.28 & 0.05 & 2.47 & 0.00 & 0.90 & 11.6 & -34.8 & -26.1 & n.d. & 1.0 & \\
\hline Z11-4-45 & 86.63 & 6.18 & 2.61 & 0.43 & 0.70 & 0.21 & 0.23 & 0.14 & 2.47 & 0.00 & 0.89 & 9.9 & -33.5 & -25.1 & -23.4 & 1.1 & \\
\hline Z11-8-5 & 95.65 & 0.18 & 0.10 & 0.15 & 0.00 & 0.08 & 0.11 & 0.09 & 2.82 & 0.00 & 0.99 & 336.4 & -39.1 & -26.0 & -25.2 & & 0.9 \\
\hline Z218 & 67.97 & 14.23 & 7.12 & 1.20 & 2.06 & 0.60 & 0.58 & 2.15 & 3.58 & 0.00 & 0.73 & 3.2 & -42.8 & -29.4 & -25.2 & & 0.8 \\
\hline Z7-1-5 & 73.98 & 9.47 & 0.81 & 0.95 & 0.01 & 0.20 & 0.20 & 2.03 & 6.24 & 0.00 & 0.86 & 7.2 & -42.0 & -26.8 & -23.6 & & 0.8 \\
\hline Z7-2-3 & 80.87 & 9.62 & 3.33 & 0.48 & 0.80 & 0.21 & 0.23 & 0.71 & 3.47 & 0.00 & 0.85 & 6.2 & -41.0 & -27.2 & -23.9 & & 0.8 \\
\hline Z9-2-1 & 82.11 & 6.27 & 0.56 & 0.69 & 0.00 & 0.24 & 0.32 & 1.12 & 4.30 & 0.00 & 0.91 & 12.0 & -39.2 & -25.3 & n.d. & & 0.9 \\
\hline $\mathrm{ZP} 1$ & 71.52 & 12.43 & 5.67 & 1.01 & 1.74 & 0.41 & 0.39 & 0.91 & 5.70 & 0.00 & 0.77 & 4.0 & -39.4 & -26.5 & -23.5 & & 0.9 \\
\hline ZT3 & 73.81 & 11.10 & 0.90 & 1.11 & 0.02 & 0.34 & 0.31 & 1.59 & 3.97 & 0.00 & 0.84 & 6.2 & -36.8 & -21.8 & n.d. & 0.8 & \\
\hline Q12-H5-11 & 58.66 & 17.43 & 9.41 & 1.23 & 2.08 & 0.40 & 0.35 & 0.66 & 8.70 & 0.00 & 0.66 & 2.2 & -30.6 & -25.9 & -23.0 & 1.3 & \\
\hline N105 & 86.89 & 5.56 & 2.15 & 0.32 & 0.36 & 0.11 & 0.08 & 1.10 & 3.25 & 0.00 & 0.91 & 11.3 & -33.0 & -23.8 & -21.8 & 1.1 & \\
\hline N14 & 90.31 & 2.12 & 0.16 & 0.00 & 0.01 & 0.00 & 0.01 & 6.08 & 1.22 & 0.00 & 0.98 & 39.6 & -32.1 & -20.5 & -20.7 & 1.2 & \\
\hline $\mathrm{N} 2-5$ & 90.56 & 5.60 & 1.63 & 0.23 & 0.31 & 0.09 & 0.10 & 0.00 & 1.38 & 0.00 & 0.92 & 12.5 & -34.3 & -25.9 & -23.8 & 1.0 & \\
\hline
\end{tabular}

Note: dryness defined as $\mathrm{C}_{1} /\left(\mathrm{C}_{1}-\mathrm{C}_{5}\right)$; n.d.: not determined. $R_{o}(\%)^{*}$ values of humic type gases were calculated based on the $\delta^{13} \mathrm{C}_{1}-R_{o}$ relationship suggested by Dai and Qi [18], $\delta^{13} \mathrm{C}_{1}=14.13 \times \log R_{o}-34.39 ; R_{o}(\%)^{* *}$ values of sapropelic-type gases were calculated according to the $\delta^{13} \mathrm{C}_{1}-R_{o}$ relationship suggested by Shen et al. [19], $\delta^{13} \mathrm{C}_{1}=40.49 \times \log R_{o}-34.00$.

4.3. Light Hydrocarbon Composition. Light hydrocarbon compositions and relevant parameters are shown in Table 1. Representative chromatograms of light hydrocarbons (samples S205 and N105) are exhibited in Figure 3. Sample S205 has higher content of $\mathrm{C}_{5}-\mathrm{C}_{7} n$-alkanes, and sample N105 has higher content of $\mathrm{C}_{6}-\mathrm{C}_{7}$ aromatics, implying different source rock kerogen types. Heptane and isoheptane values are commonly used to identify the maturity of gas and oil [3]. Due to the enhanced contents of alkanes in $\mathrm{C}_{7}$ hydrocarbons from the saline lacustrine environment in the western Qaidam Basin [36], it is not suitable to study the maturity of gas by using heptane and isoheptane values.

A cross plot of $n-\mathrm{C}_{7} / \mathrm{MCC}_{6}$ and toluene $/ n-\mathrm{C}_{7}$ is often used to study the secondary alternation of petroleum in a reservoir [37]. Figure 4 shows that most gases in the western
Qaidam Basin are in the area of the original, and some experienced secondary alternations. YII5-13, HN5-43-3, and Q12-H5-11 gas samples may have suffered biodegradation, and $\delta^{13} \mathrm{C}_{1}$ values of YII5-13 and HN5-43-3 are $-47.7 \%$ and $-54.6 \%$, respectively, implying that these light hydrocarbons were from the bacterial decomposition of oil.

\section{Discussion}

5.1. Genetic Types of Natural Gases. A plot of $\delta^{13} \mathrm{C}_{1}$ vs. $\mathrm{C}_{1} /\left(\mathrm{C}_{2}\right.$ $+\mathrm{C}_{3}$ ) is widely applied to identify kerogen types for gas generation $[13,38]$. Figure 5 shows that most gases in the western Qaidam Basin are in the thermogenic area and a trace of them are close to the type-II kerogen area, some are distributed in or near the type-III kerogen area, and several fall 


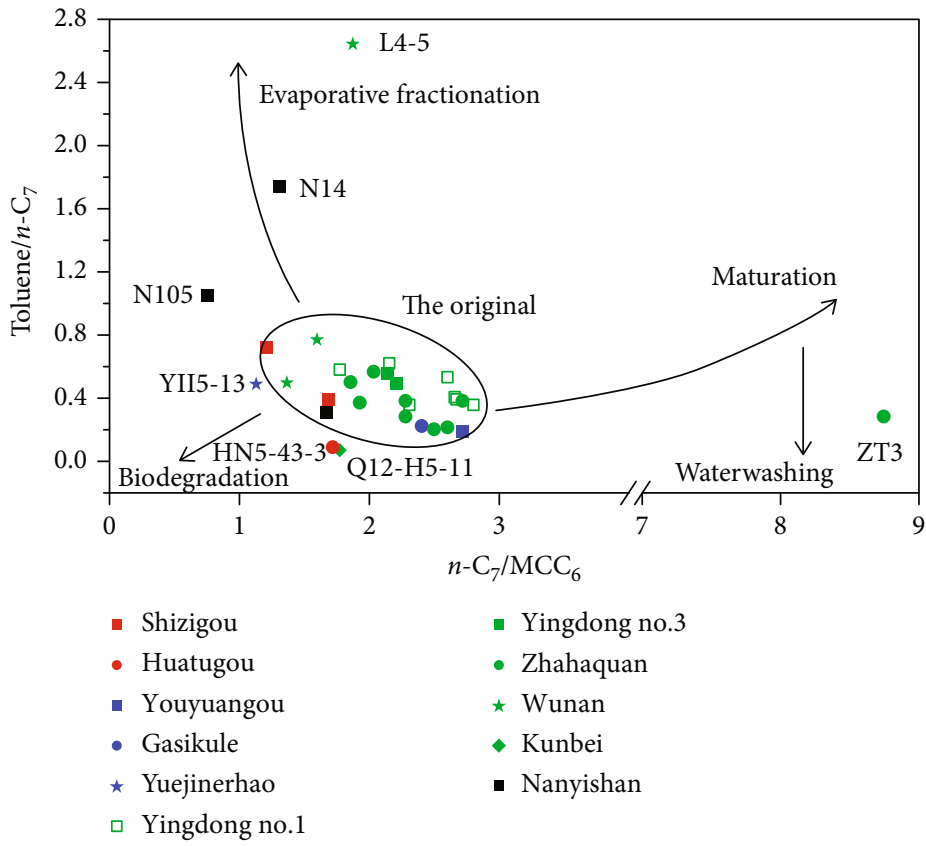

Figure 4: A cross plot of $n-\mathrm{C}_{7} / \mathrm{MCC}_{6}$ vs. toluene $/ n-\mathrm{C}_{7}$ of gases in the western Qaidam Basin (modified after [37]).

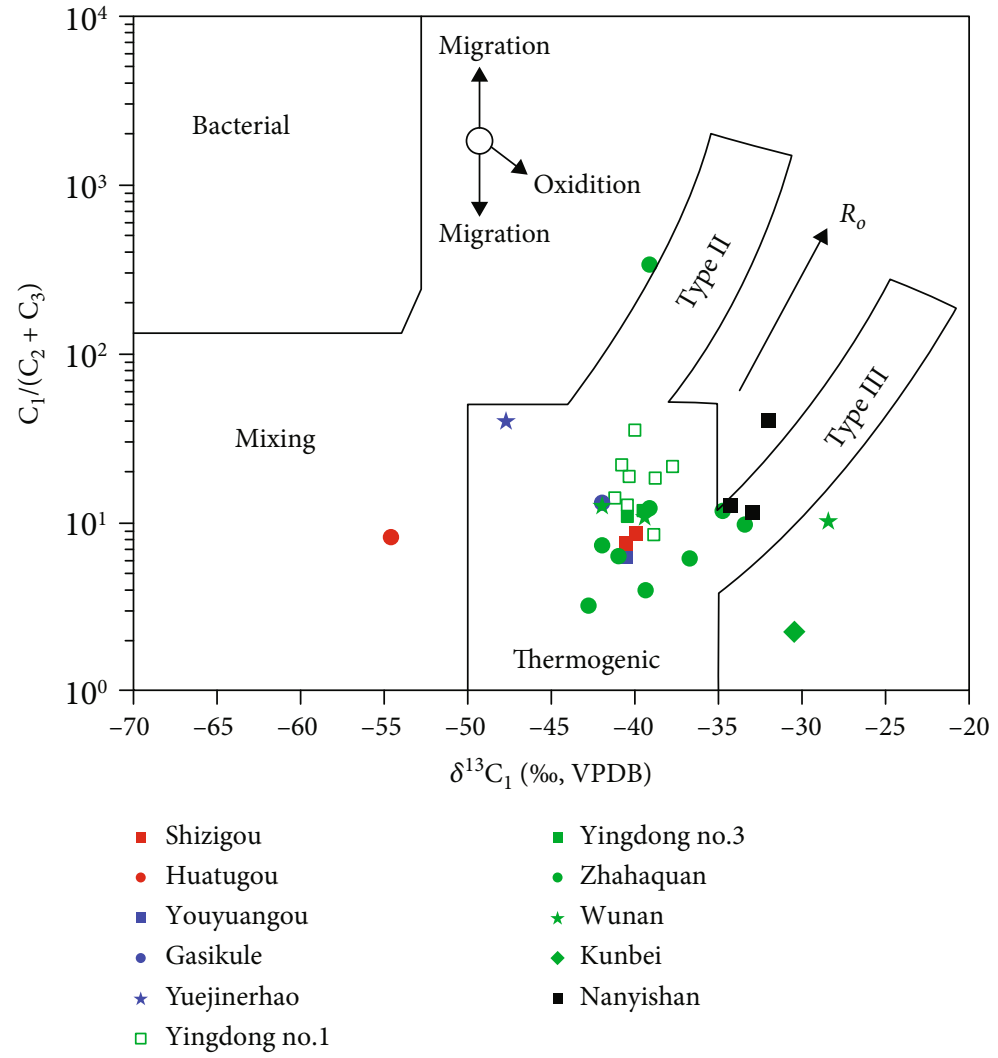

Figure 5: Diagram of $\mathrm{C}_{1} /\left(\mathrm{C}_{2}+\mathrm{C}_{3}\right)$ vs. $\delta^{13} \mathrm{C}_{1}$ of gases in the western Qaidam Basin (modified after $[13,38]$ ).

in the area of mixing. The result indicates that most gases in the western Qaidam Basin were generated from sapropelic source rocks, while several gases in the Zhahaquan, Wunan, Kunbei, and Nanyishan fields were derived from humic source rocks, for example, W8-11, Z11-33-5, Z11-4-5, Q12$\mathrm{H} 5-11, \mathrm{~N} 14, \mathrm{~N} 105$, and N2-5. Furthermore, $\delta^{13} \mathrm{C}_{2}$ is often used as an identification of different types of gas. Zhang et al. [17] proposed a $\delta^{13} \mathrm{C}_{2}>-23.0 \%$ cutoff for coal-type 
gas and a $\delta^{13} \mathrm{C}_{2}<-24.8 \%$ cutoff for oil-type gas according to a research on the saline lacustrine natural gas in western Qaidam Basin. It is believed that most gases in the western Qaidam Basin are oil-type gases; W8-11, Z11-33-5, Z11-45, Q12-H5-11, ZT3, N14, and N105 are most likely the coal-type gases.

Relative abundance of $n$-heptane $\left(n-\mathrm{C}_{7}\right)$, methylcyclohexane $\left(\mathrm{MCC}_{6}\right)$, and dimethylcyclopentane $\left(\mathrm{DMCC}_{5}\right)$ may be used to identify the type of gases $[6,7]$. Previous studies show that $n-\mathrm{C}_{7}$ is predominantly derived from algae and bacteria; $\mathrm{MCC}_{6}$ is primarily from lignin, cellulose, and components of higher plants; and $\mathrm{DMCC}_{5}$ of various structures is mainly derived from the steroid and terpenoid compounds of aquatic organisms [2, 39]. Most gases in the western Qaidam Basin plot are in the sapropelic organic matter area, indicating that gases were mainly generated from sapropelic source rock (Figure 6). Only one gas sample, N105, falls in the humic organic matter area, implying that this gas was probably derived from humic source rock. Several gas samples have high contents of $\Sigma \mathrm{DMCC}_{5}$, which attributes to strong depletion of $n-\mathrm{C}_{7}$ from biodegradation.

Different genetic types obtained by $\mathrm{C}_{1}-\mathrm{C}_{3}$ fractions and $\mathrm{C}_{7}$ fraction were observed in several gases in the Nanyishan and Zhahaquan fields of western Qaidam Basin. The $\delta^{13} C_{1}$ reveals that oil-type gas was generated at equivalent vitrinite reflectance $\left(R_{o}\right)$ of $0.6-0.9 \%$ with an average of $0.8 \%$ and that coal-type gas was generated in the range of $0.8-1.5 \%$ with an average of $1.1 \%$, which will be discussed below. Pyrolysis of source rocks shows that sapropelic kerogens are predominant in the Qaidam Basin, and also develops some humic kerogens $[32,40]$. In contrast to the sapropelic source rock at the moderately mature stage, humic source rock at the late mature stage would generate more $\mathrm{C}_{1}-\mathrm{C}_{3}$ hydrocarbons and fewer $\mathrm{C}_{7}$ hydrocarbons. Besides, a previous study proposed that coal-type gas in the Nanyishan field was derived from the coal measures of the Jurassic formation with a higher maturity [41], so there are probably contributions of coal-type gas from the Jurassic source rock in other fields in the western Qaidam Basin. Thus, it is inferred that mixed gas originated from the humic organic matter in deeply buried strata with an average $R_{o}$ value of $1.1 \%$ and the sapropelic organic matter in the Tertiary deposition with an average $R_{o}$ value of $0.8 \%$.

Overall, based on the genetic type obtained from $\mathrm{C}_{1}-\mathrm{C}_{3}$ and $\mathrm{C}_{7}$ hydrocarbons, four types of gases are identified: coal-type gas, featured by an enriched $\delta^{13} \mathrm{C}_{2}$ value and high $\mathrm{MCC}_{6}$ content; oil-type gas, characterized by a depleted $\delta^{13} \mathrm{C}_{2}$ value and low $\mathrm{MCC}_{6}$ content; biodegraded gas, featured by low $n-\mathrm{C}_{7}$ content; and mixed gas, characterized by a relatively enriched $\delta^{13} \mathrm{C}_{2}$ value and low $\mathrm{MCC}_{6}$ content. Oil-type gas is the predominant-type gas in the western Qaidam Basin; coal-type gas is mainly distributed in the Zhahaquan and Nanyishan fields; mixed gas is mainly in the Zhahaquan, Wunan, and Nanyishan fields; and biodegraded gas is distributed in the Huatugou and Yuejinerhao fields.

\subsection{Maturity of Natural Gas}

5.2.1. Stable Carbon Isotope of Methane. Natural gas maturity is closely related to gas-source correlation, and maturity of source rock could be estimated with the empirical relationships between $\delta^{13} C_{1}$ and $R_{o}[18,19]$. Because of different types of natural gases in the western Qaidam Basin, maturities of gases were calculated by the $\delta^{13} \mathrm{C}_{1}-R_{o} \%$ relationships: Equation (1) for coal-type gas [18] and Equation (2) for oiltype gas [19].

$$
\begin{aligned}
& \delta^{13} \mathrm{C}_{1}=14.13 \log \left(R_{o}-34.39\right), \\
& \delta^{13} \mathrm{C}_{1}=40.49 \log \left(R_{o}-34.00\right) .
\end{aligned}
$$

Calculated thermal maturities of gases in the western Qaidam Basin are exhibited in Table 3. The $R_{o}$ values for oil-type gases range from $0.6 \%$ to $0.9 \%$, with an average of $0.8 \%$, indicating that most gases are moderately mature, which matched well with the real thermal maturity of the Tertiary source rocks. The $R_{o}$ values for coal-type gases are in the range of $0.8-1.5 \%$, with an average of $1.1 \%$, implying that coal-type gases reach mature and highly mature stages.

\subsubsection{Chemical Composition of Heptane. The GTMRH} obtained from light hydrocarbons was proposed by Mango [42] and modified by BeMent et al. [21] and Mango [8]. They suggested that the ratio of 2,4-dimethylpentane to 2,3-dimethylpentane $\left(2,4-\mathrm{DMC}_{5} / 2,3-\mathrm{DMC}_{5}\right)$ was a function of temperature, independent of time and kerogen types. The following empirically derived formula Equation (3) was published to calculate the GTMRH using $\mathrm{C}_{7}$ light hydrocarbons [8].

$$
T\left({ }^{\circ} \mathrm{C}\right)=140+15 \ln \left(\frac{2,4-\mathrm{DMC}_{5}}{2,3-\mathrm{DMC}_{5}}\right)
$$

Based on the relationship, the GTMRH in the western Qaidam Basin are calculated and exhibited in Table 1. GTMRH positively correlated with the $\delta^{13} \mathrm{C}_{1}$ and not significantly, probably because of the influence of the saline lacustrine sedimentary environment or the mixed sources from different organic matter. But gas samples in the Yingdong field show positive correlations between GTMRH and $\delta^{13} \mathrm{C}_{1}$ which are most likely because they have same/similar sources (Figure $7(\mathrm{a})$ ). Furthermore, GTMRH is positively correlated with ratio of 2-methylhexane to 3-methylhexane $\left(2-\mathrm{MC}_{6} / 3-\mathrm{MC}_{6}\right)$ which increases with increasing maturity (Figure 7(b)) [42].

GTMRH data reveal that the maturity of gases in the Yingdong, Zhahaquan, Wunan, and Nanyishan fields is higher than that in the Shizigou, Huatugou, Youyuangou, Gasikule, and Yuejinerhao fields (Figure 8). The $\delta^{13} \mathrm{C}_{1}$ and gas dryness indicate higher maturity of gases in the former fields. Overall, maturity of gas increases from west to east in the southwestern Qaidam Basin. This is most likely because major source rocks near the Yingdong, Zhahaquan, and Nanyishan fields have higher maturity [43]. Moreover, most faults became increasingly active since the late Eocene, and these faults extend into the depression and form connections between the source rocks and the reservoir groups, which allow oil and gas with higher maturity to 


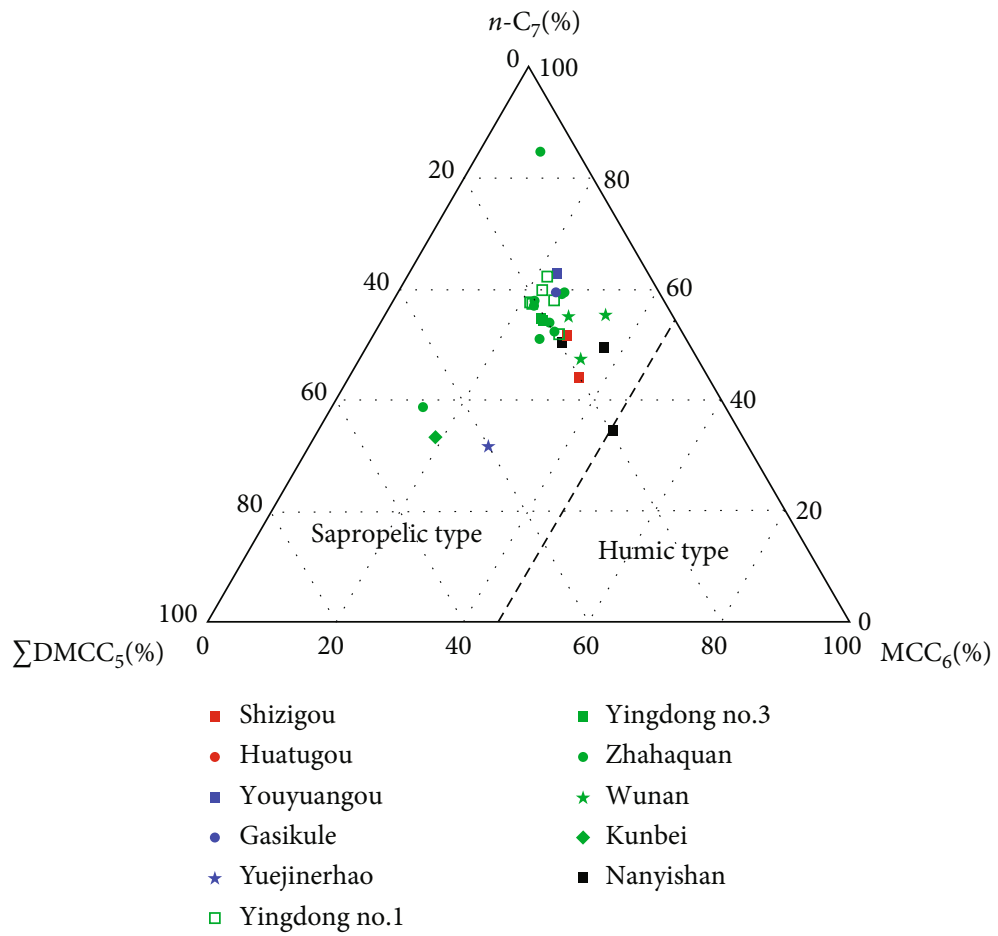

Figure 6: Ternary diagram of $\mathrm{C}_{7}$ series in natural gases from the western Qaidam Basin.

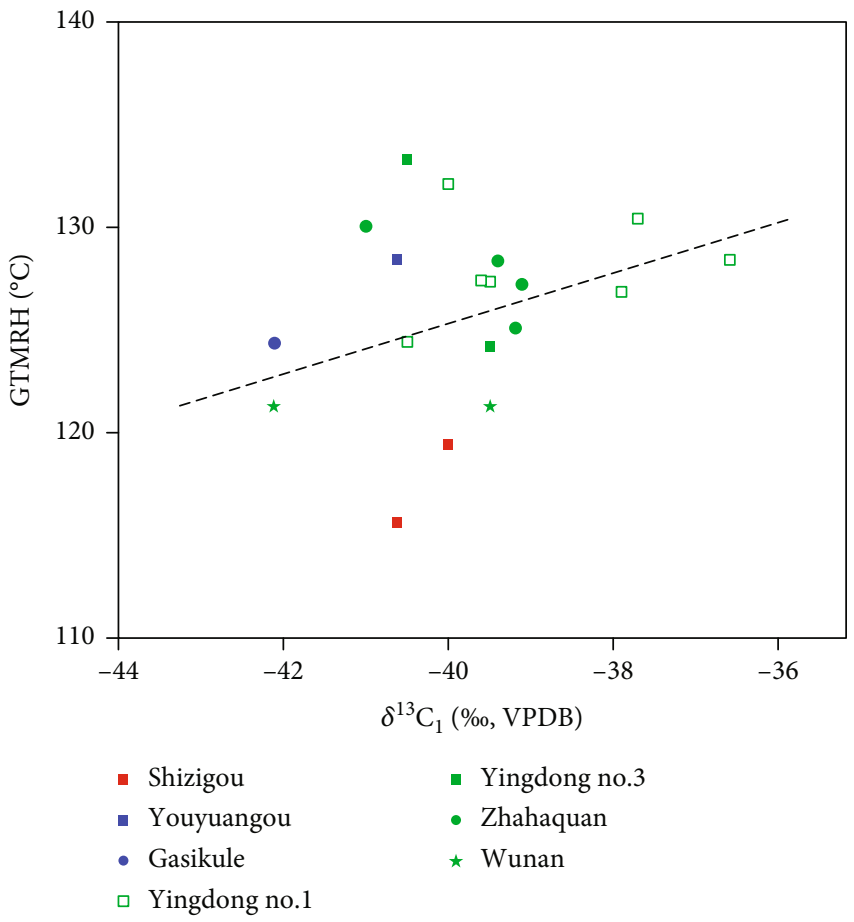

(a)

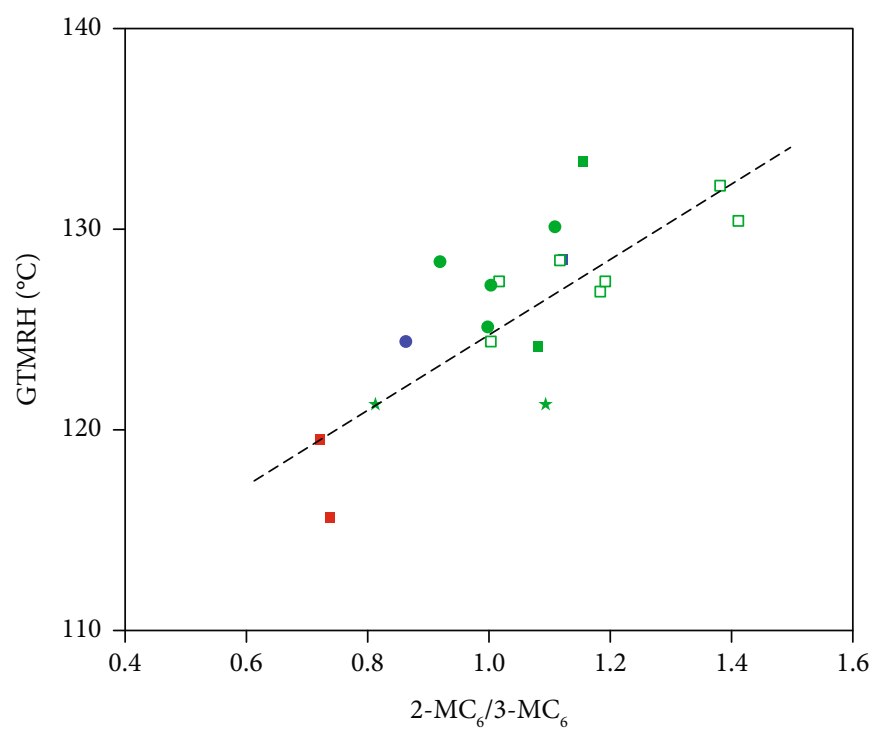

Figure 7: Cross plots of (a) GTMRH vs. $\delta^{13} \mathrm{C}_{1}$ and (b) GTMRH vs. 2-MC $\mathrm{MC}_{6} / 3-\mathrm{MC}_{6}$ for single sourced gases in the western Qaidam Basin.

migrate along the faults to reservoirs in midlevel and shallow formations [44]. Therefore, the maturity of gas in the Yingdong, Zhahaquan, Wunan, and Nanyishan fields is higher than that in others.
5.2.3. Application of Generation Temperature of Major Reservoired Hydrocarbons to Petroleum Charge History. Most studies suggest that there are primarily two petroleum charging episodes in the western Qaidam Basin [25-30]. The early 


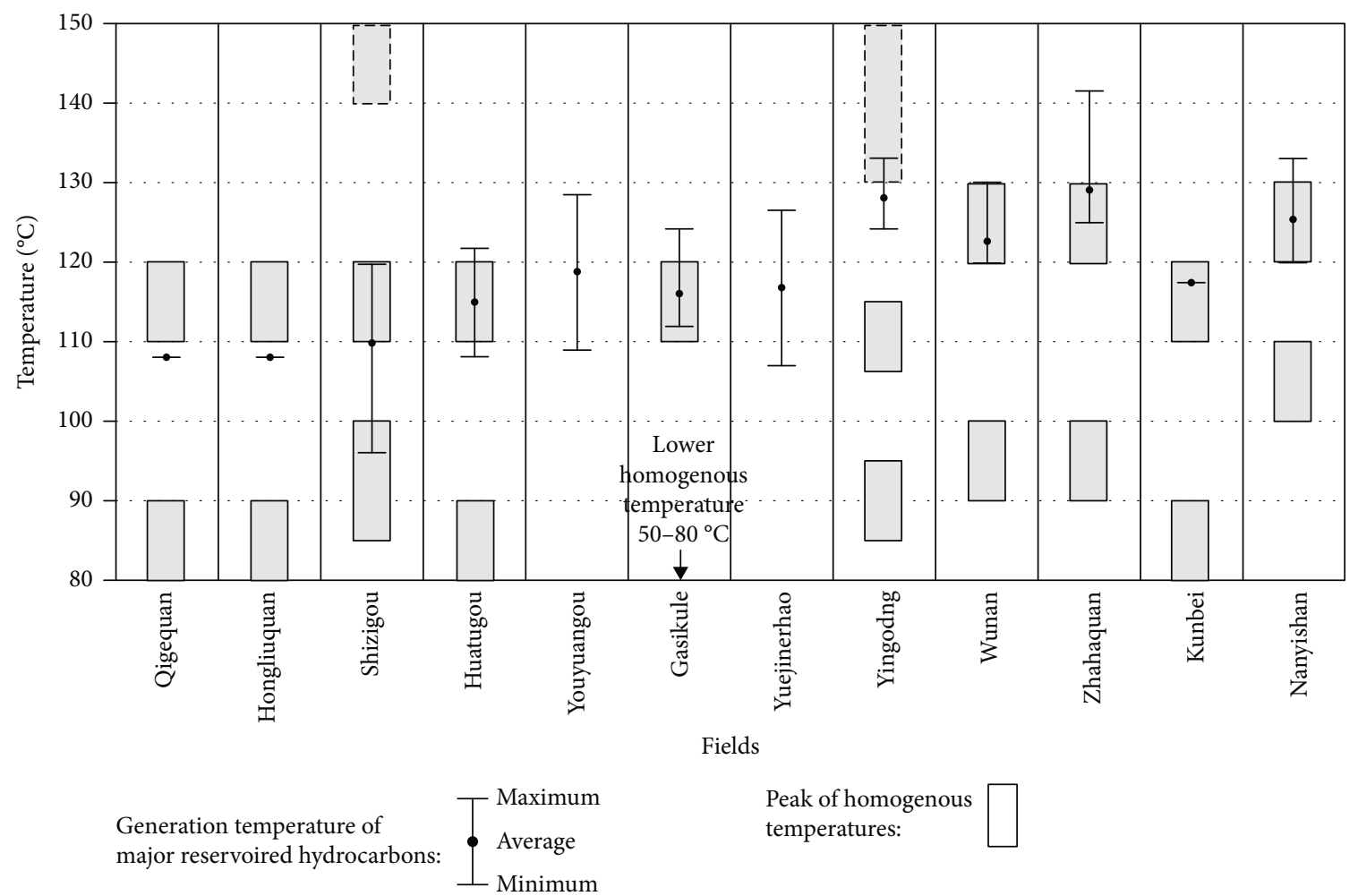

FIGURE 8: Relationship between generation temperature of major reservoired hydrocarbons (GTMRH) and peaks of homogenization temperature in different oil fields in the western Qaidam Basin. Supplementary GTMRH data for the Qigequan, Hongliuquan, Shizigou, Huatugou, Youyuangou, Gasikule, and Yuejinerhao oil fields are from Zhu et al. [31]. Peak homogenous temperature of fluid inclusion data for the Qigequan and Hongliuquan fields are from Fu et al. [1], Shizigou is from Li et al. [25], Huatugou is from Liu et al. [26], Gasikule and Nanyishan are from Li et al. [27], Yingdong is from Sui et al. [28], Zhahaquan is from Wang et al. [29], and Kunbei is from Chen et al. [30]. Solid border of the peak homogenous temperature is presented in published articles; the dotted border peak is based on latest studies.

charge occurred in the early Shangyoushashan period (about $10 \mathrm{Ma}$ ), and the later charge happened during the Shizigou period to the present (about 4-0 Ma), both of which matched well with the middle and late periods of the Himalayan movement [45]. In this study, all gas samples were collected from reservoired petroleum which was the result of effective hydrocarbon accumulation during the major charging episode. Hence, GTMRH obtained from the $\mathrm{C}_{7}$ light hydrocarbon might be related to homogenous temperatures and could be used to study major petroleum charging episodes.

Combining with burial history (Figure 9), it shows that the early charge occurred in the early Shangyoushashan period with peak temperatures of $80-100^{\circ} \mathrm{C}$, and the later charge happened during the Shizigou period to the present with peak temperatures of $110-130^{\circ} \mathrm{C}$ in the western Qaidam Basin (Figure 8). Both peak temperatures reveal that the maturity of petroleum in the Yingdong, Wunan, and Zhahaquan fields is higher than that in other fields in southwestern Qaidam Basin, which is in agreement with the study above. Figure 8 displays that GTMRH have a close relation with peak temperatures in the western Qaidam Basin. GTMRH in the Huatugou, Gasikule, Wunan, Zhahaquan, Kunbei, and Nanyishan fields overlap with higher peak temperatures, and their average GTMRH are in the range of higher ones, implying that the major petroleum charging episode is related to the later period. In the Qigequan and Hongliuquan fields, GTMRH are about $108^{\circ} \mathrm{C}$, which are close to higher peak temperatures $\left(110-120^{\circ} \mathrm{C}\right)$, indicating that the major petroleum charging episode is related to the later period, too. Though there is no homogenous temperature in the Youyuangou field, GTMRH indicates that petroleum was generated at a relatively higher mature stage. The GTMRH in the Shizigou field range from $96^{\circ} \mathrm{C}$ to $116^{\circ} \mathrm{C}$, overlapping with lower and higher peak temperatures, and the average GTMRH value is more close to the higher one, suggesting that the major petroleum charging episode is related to the later period. Furthermore, the latest study has found higher homogeneous temperatures ranging from $140^{\circ} \mathrm{C}$ to $150^{\circ} \mathrm{C}$ in the Shizigou field. GTMRH in the Yingdong field are higher than both peak temperatures, and recent research suggested a petroleum charge event with homogeneous temperatures greater than $130^{\circ} \mathrm{C}$. It may suggest an abundant amount of petroleum migration into shallow reservoirs due to strong tectonic activities during the late period of the Himalayan movement. On the whole, the GTMRH obtained from the $\mathrm{C}_{7}$ light hydrocarbon could reflect a major petroleum charging episode, implying that a later charge of petroleum is predominant in the western Qaidam Basin. 


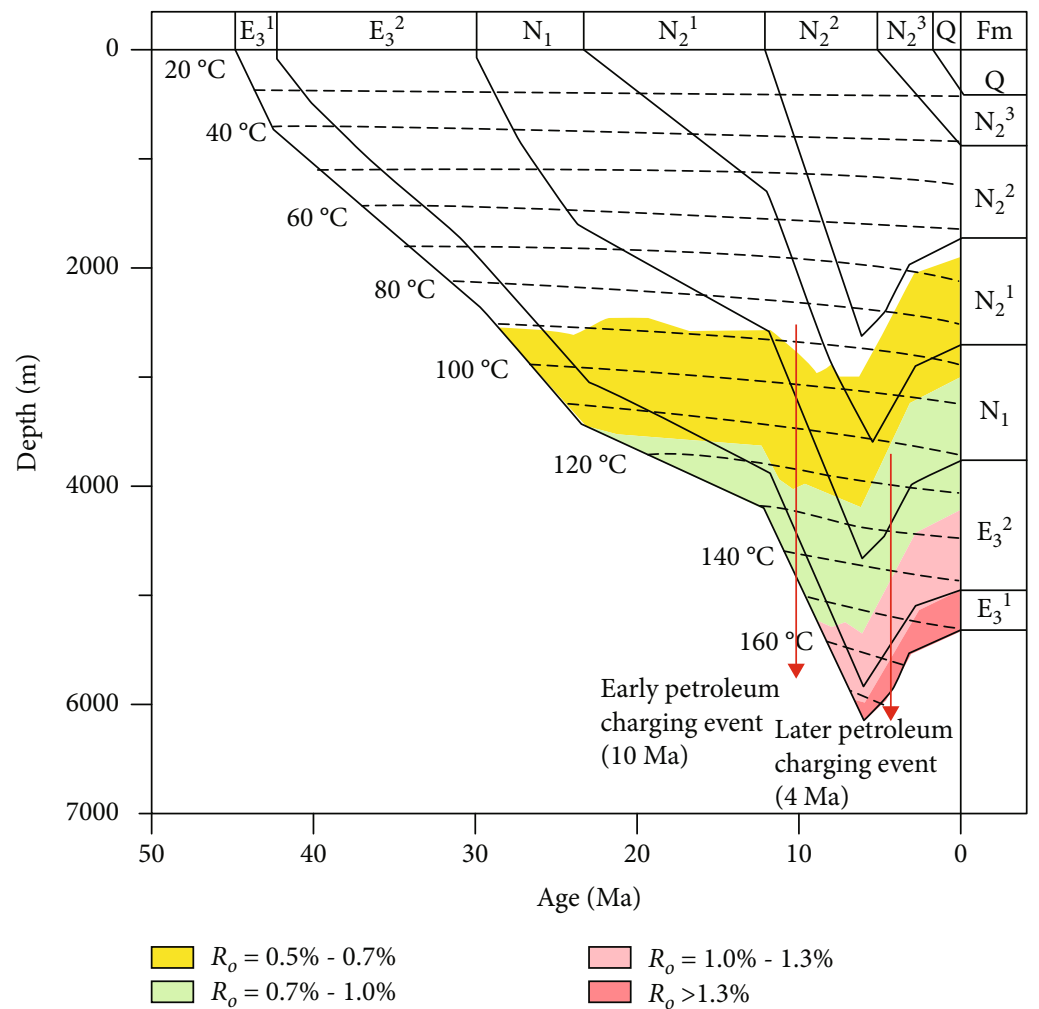

FIGURE 9: Sedimentary burial profile for the Yingdong field in the western Qaidam Basin (modified after [47]), and burial history is similar with most fields in the western Qaidam Basin.

The GTMRH calculated from the $\mathrm{C}_{7}$ light hydrocarbon are mainly greater than $100^{\circ} \mathrm{C}$, indicating that petroleum was mainly generated from the Tertiary source rocks at the middle stage of the oil window, consistent with the conclusion achieved from the $\delta^{13} \mathrm{C}_{1}$ study. It is revealed from the light hydrocarbon study that if different molecular weight hydrocarbons in most reservoirs came from a similar origin, then petroleum in the western Qaidam Basin was primarily generated at the middle stage of the oil window and accumulated in the reservoirs during the late period of the Himalayan movement.

5.3. Implications for Hydrocarbon Exploration. The study above reveals that there are deep hydrocarbon fluid sources in the Shizigou, Yingdong, Zhahaquan, and Nanyishan fields. The GTMRH calculated from the $\mathrm{C}_{7}$ light hydrocarbon indicates that petroleum accumulation mainly occurred during the late period of the Himalayan movement (about $4 \mathrm{Ma}$ ), simultaneous with the main oil-generation stage. Previous studies show that strong tectonic movement in the western Qaidam Basin leads to the formation of many deep faults and anticlinal traps [26, 44], which allows petroleum to migrate to traps along the faults. Thus, abundant petroleum accumulated in the midshallow layers and has been explored, and it is most likely to have a high exploration potential in deep formation. Besides, in the western Qaidam Basin, oil and gas were mainly derived from sapropelic source rocks of the Tertiary depositions and also some contributions of coal-type gas from the Tertiary and probably the Jurassic source rocks. In summary, deep reservoirs of paleouplifts adjacent to the hydrocarbon-generating depressions are estimated as a favorable area for further exploration in the western Qaidam Basin.

\section{Conclusions}

The gas composition of light hydrocarbons $\left(\mathrm{C}_{5}-\mathrm{C}_{7}\right)$ and carbon isotopes was analyzed from 31 gases in the western Qaidam Basin. The genetic type and thermal maturity of natural gas were determined by $C_{1}-C_{3}$ and $C_{5}-C_{7}$ hydrocarbons. The generation temperature of major reservoired hydrocarbons was calculated from the $\mathrm{C}_{7}$ light hydrocarbon and was applied to petroleum charge history. The following main conclusions have been reached:

(1) Four types of gases are identified: oil-type gas, coaltype gas, biodegraded gas, and mixed gas. The oiltype gas is the predominant-type gas in the western Qaidam Basin; coal-type gas is mainly distributed in the Zhahaquan and Nanyishan fields; mixed gas is mainly in the Zhahaquan, Wunan, and Nanyishan fields; and biodegraded gas is mainly distributed in the Huatugou and Yuejinerhao fields

(2) The $R_{o}$ values of gas range from $0.6 \%$ to $1.5 \%$, with an average value of $0.9 \%$. The GTMRH calculated from the $\mathrm{C}_{7}$ components range from $115.6^{\circ} \mathrm{C}$ to $141.7^{\circ} \mathrm{C}$, with an average value of $126.5^{\circ} \mathrm{C}$. Both maturity 
indicators have relatively positive correlation and reveal that the maturity of gas increases from west to east in the southwestern Qaidam Basin

(3) The major petroleum charge episode in the western Qaidam Basin, which is during the late period of the Himalayan movement, is identified based on GTMRH combining with the homogenous temperature of petroleum inclusions

(4) Deep reservoirs of paleouplifts adjacent to the hydrocarbon-generating depressions are estimated as a favorable area for further exploration in the western Qaidam Basin

\section{Data Availability}

The experimental data used to support the findings of this study are included within the article.

\section{Conflicts of Interest}

The authors declare that there are no conflicts of interest.

\section{Acknowledgments}

This study was supported by the National Science and Technology Projects of Ministry of Science and Technology of China (grant number 2016ZX05003002-004) and National Nature Science Foundation of China (grant numbers 41072105 and 41872147).

\section{References}

[1] S. T. Fu, J. Y. Yuan, L. Q. Wang et al., Study on Geologic Characteristics and Hydrocarbon Accumulation in Qaidam Basin, Science Press, Beijing, China, 2014.

[2] D. Leythaeuser, R. G. Schaefer, C. Cornford, and B. Weiner, "Generation and migration of light hydrocarbons (C2-C7) in sedimentary basins," Organic Geochemistry, vol. 1, no. 4, pp. 191-204, 1979.

[3] K. F. M. Thompson, "Classification and thermal history of petroleum based on light hydrocarbons," Geochimica et Cosmochimica Acta, vol. 47, no. 2, pp. 303-316, 1983.

[4] J. M. Hunt, "Generation and migration of light hydrocarbons," Science, vol. 226, no. 4680, pp. 1265-1270, 1984.

[5] F. D. Mango, "Transition metal catalysis in the generation of petroleum and natural gas," Geochimica et Cosmochimica Acta, vol. 56, no. 1, pp. 553-555, 1992.

[6] J. X. Dai, "Identification of coal formed gas and oil type gas by light hydrocarbons," Petroleum Exploration and Development, vol. 20, pp. 26-32, 1993.

[7] G. Y. Hu, J. Li, J. Li et al., "A discussion on discrimination of natural gas' source by light hydrocarbon," Science in China (series D), vol. 37, Suppl. II, pp. 111-117, 2007.

[8] F. D. Mango, "The light hydrocarbons in petroleum: a critical review," Organic Geochemistry, vol. 26, no. 7-8, pp. 417-440, 1997.

[9] B. Carpentier, P. Ungerer, I. Kowalewski, C. Magnier, J. P. Courcy, and A. Y. Huc, "Molecular and isotopic fractionation of light hydrocarbons between oil and gas phases," Organic Chemistry, vol. 24, pp. 1115-1139, 1996.

[10] Q. Liu, X. Wu, X. Wang et al., "Carbon and hydrogen isotopes of methane, ethane, and propane: a review of genetic identification of natural gas," Earth-Science Reviews, vol. 190, pp. 247-272, 2019.

[11] Y. C. Xu, Genetic Theories of Natural Gases and their Application, Science Press, Beijing, 1994.

[12] J. X. Dai, "Identification and distinction of various alkane gases," Science China, vol. 35, pp. 1246-1257, 1992.

[13] B. B. Bernard, J. M. Brooks, and W. M. Sackett, "Light hydrocarbons in recent Texas continental shelf and slope sediments," Journal of geophysical research planets, vol. 83, no. C8, pp. 4053-4061, 1978.

[14] J. X. Dai, S. F. Qin, S. Z. Tao, G. Y. Zhu, and J. K. Mi, "Development trends of natural gas industry and the significant progress on natural gas geological theories in China," Natural Gas Geoscience, vol. 16, pp. 127-142, 2005.

[15] A. A. Prinzhofer and A. Y. Huc, "Genetic and post-genetic molecular and isotopic fractionations in natural gases," Chemical Geology, vol. 126, no. 3-4, pp. 281-290, 1995.

[16] M. J. Kotarba and K. Nagao, "Composition and origin of natural gases accumulated in the Polish and Ukrainian parts of the Carpathian region: gaseous hydrocarbons, noble gases, carbon dioxide and nitrogen," Chemical Geology, vol. 255, no. 3-4, pp. 426-438, 2008.

[17] X. B. Zhang, Y. Hu, L. Y. Ma et al., "Carbon isotopic characteristics, genesis and distribution of the natural gas of the Tertiary saline lake in the western part of Qaidam Basin," Science in China (Series D), vol. 32, pp. 598-608, 2002.

[18] J. X. Dai and H. F. Qi, "Relationship of $\delta^{13} \mathrm{C}-\mathrm{R}_{\mathrm{o}}$ of coal-derived gas in China," China Science Bulletin, vol. 34, pp. 690-692, 1989.

[19] P. Shen, Y. Xu, X. Wang, D. Liu, Q. Shen, and W. Liu, Studies on Geochemical Characteristics of Gas Source Rocks and Natural Gas and Mechanism of Genesis of Gas, Gansu Science and Technology Press, Lanzhou, 1991.

[20] V. Dieckmann, P. G. Caccialanza, and R. Galimberti, "Evaluating the timing of oil expulsion: about the inverse behaviour of light hydrocarbons and oil asphaltene kinetics," Organic Geochemistry, vol. 33, no. 12, pp. 1501-1513, 2002.

[21] W. O. BeMent, R. A. Levey, and F. D. Mango, “The temperature of oil generation as defined with $\mathrm{C}_{7}$ chemistry maturity parameter (2,4-DMP/2,3-DMP ratio)," in Organic Geochemistry: Developments and Applications to Energy, Climate, J. O. Grimalt and C. Dorronsoro, Eds., pp. 505-507, Environment and Human History, Donostia, San Sebastian, Spain, 1995.

[22] H. M. Chung, C. C. Walters, S. Buck, and G. Bingham, "Mixed signals of the source and thermal maturity for petroleum accumulations from light hydrocarbons: an example of the Beryl field," Organic Geochemistry, vol. 29, no. 1-3, pp. 381-396, 1998.

[23] Y. M. Zhu, "Calculation of hydrocarbon-generating temperature and its application to exploration," Experimental Petroleum Geology, vol. 22, no. 2, pp. 167-171, 2000.

[24] D. M. Jarvie, "Williston Basin petroleum systems: inferences from oil geochemistry and geology," Mountain Geologist, vol. 38, pp. 19-41, 2001.

[25] H. Li, D. Z. Tang, H. Xu, and F. L. Li, "Analysis of hydrocarbon accumulation period in Paleogene reservoirs, Shizigou oil field 
of Qaidam Basin," Petroleum Geology and Recovery Efficiency, vol. 20, pp. 30-36, 2013.

[26] Z. Liu, Y. Q. Dang, H. Y. Li, and X. Z. Gao, "Characteristics of the late hydrocarbon accumulation," Journal of Xi'an Shiyou University (Natural Science Edition), vol. 22, pp. 1-6, 2007.

[27] X. Q. Li, N. N. Zhong, K. D. Wang et al., "Study on Tertiary oil charges to typical structures in western Qaidam Basin," Xinjiang Petroleum Geology, vol. 32, pp. 1-3, 2011.

[28] L. W. Sui, S. H. Fang, Y. H. Sun et al., "The tectonic evolution and accumulation controlling characteristics of ShizigouYingdong structural belt of western Qaidam Basin," Earth Science Frontiers, vol. 21, pp. 261-270, 2014.

[29] L. Wang, H. Wu, K. Wang et al., "Hydrocarbon charge history of Zhahaquan area, western Qaidam Basin," Science Technology and Engineering, vol. 34, pp. 202-209, 2017.

[30] S. J. Chen, J. G. Lu, D. D. Ma et al., "Origin and accumulation characteristics of the oil from hanging walls of Kunbei faultterrace belt in Qaidam Basin," Acta Petrolei Sinica, vol. 33, pp. 915-924, 2012.

[31] Y. M. Zhu, H. X. Weng, A. G. Su, D. G. Liang, and D. H. Peng, "Geochemical characteristics of Tertiary saline lacustrine oils in the Western Qaidam Basin, Northwest China," Applied Geochemistry, vol. 20, pp. 1875-1889, 2005.

[32] A. D. Hanson, B. D. Ritts, D. Zinniker, J. M. Moldowan, and U. Biffi, "Upper Oligocene lacustrine source rocks and petroleum systems of the northern Qaidam Basin, Northwest China," American Association of Petroleum Geologists Bulletin, vol. 85, pp. 601-619, 2001.

[33] D. F. Huang, J. C. Li, D. J. Zhang, X. M. Huang, and Z. L. Zhou, "Maturation sequence of Tertiary crude oils in the Qaidam basin and its significance in petroleum resource assessment," Journal of Southeast Asian Earth Sciences, vol. 5, pp. 359366, 1991.

[34] J. P. Bao, C. S. Zhu, and L. Q. Wang, "Geochemical characteristic comparison of crude oil samples from the western Qaidam Basin," Oil and Gas Geology, vol. 31, pp. 353-359, 2010.

[35] N. K. Cañipa-Morales, C. A. Galán-Vidal, M. A. GuzmánVega, and D. M. Jarvie, "Effect of evaporation on $\mathrm{C}_{7}$ light hydrocarbon parameters," Organic Geochemistry, vol. 34, no. 6, pp. 813-826, 2003.

[36] P. R. Wang, G. J. Xu, T. R. Xiao, and D. J. Zhang, “Application of $\mathrm{C}_{7}$ light hydrocarbon parameters in identifying source rock depositional environment," Petroleum Exploration and Development, vol. 34, pp. 156-159, 2007.

[37] K. F. M. Thompson, "Fractionated aromatic petroleums and the generation of gas-condensates," Organic Geochemistry, vol. 11, no. 6, pp. 573-590, 1987.

[38] M. J. Whiticar, "Carbon and hydrogen isotope systematics of bacterial formation and oxidation of methane," Chemical Geology, vol. 161, no. 1-3, pp. 291-314, 1999.

[39] J. L. Clayton, D. D. Rice, and G. E. Michael, "Oil-generating coals of the San Juan Basin, New Mexico and Colorado, U.S.A.," Organic Geochemistry, vol. 17, no. 6, pp. 735-742, 1991.

[40] B. Zhang, Y. Y. He, Y. Chen, Q. Y. Meng, and L. Yuan, "Geochemical characteristics and oil accumulation significance of the high quality saline lacustrine source rocks in the western Qaidam Basin, NW China," Acta Petrolei Sinica, vol. 38, pp. 1158-1167, 2017.

[41] G. M. Tang, Q. Luo, X. Q. Pang, C. F. Xiang, L. Q. Wang, and H. Y. Wei, "Origin and accumulation model of natural gas in
Nanyishan structure of western Qaidam Basin," Aca Petrolei Sinica, vol. 36, pp. 446-456, 2015.

[42] F. D. Mango, "An invariance in the isoheptanes of petroleum," Science, vol. 273, pp. 514-517, 1987.

[43] Y. S. Zhang, F. Zhou, B. Wang, X. Zeng, J. Zhang, and X. B. Zhang, "Genesis, type and reservoir formation law of natural gas in western Qaidam Basin," China Petroleum Exploration, vol. 24, pp. 498-508, 2019.

[44] L. Wu, A. C. Xiao, D. D. Ma et al., "Cenozoic fault systems in southwest Qaidam Basin, northeastern Tibetan Plateau: geometry, temporal development, and significance for hydrocarbon accumulation," AAPG Bulletin, vol. 98, pp. 1212-1234, 2014.

[45] Y. Q. Dang, J. H. Xiong, Z. Liu, D. D. Ma, L. Q. Wang, and W. L. Li, "Main factors controlling hydrocarbon accumulation in Qaidam basin," Oil and Gas Geology, vol. 25, pp. 614-619, 2004.

[46] D. H. Peng, Geology, geochemical characteristics and mechanism of hydrocarbon-generating for source rocks from the Tertiary salty lacustrine facies in the west region of the Qaidam Basin, Guangzhou Institute of Geochemistry, Chinese Academy of Science (Ph.D. thesis, in Chinese with English abstract), 2004.

[47] Q. Y. Meng, L. L. Gui, Y. Chen et al., "Hydrocarbon charging history of typical oilfields in southwestern Qaidam Basin," Natural Gas Geoscience, vol. 26, pp. 252-260, 2015. 